\title{
Detrusor Response to Outlet Obstruction
}

\author{
Edward J. McGuire \\ Section of Urology, Department of Surgery, University of Michigan Medical Center, Ann Arbor, Michigan 48109, USA
}

Summary. Bladder responses to outlet obstruction are thought to involve muscular hyperplasia, increased pressure, and finally, decompensation. While these things probably do occur, the process of bladder response to obstruction also includes loss of reflex control and deposition of collagen.

The final result of obstructive uropathy, detrusor decompensation, with a large fibrotic bladder and a poor flow rate, residual urine, dilated ureters, and impaired renal function or total urinary retention, would probably be accepted as such by most urologists, and students of urodynamics. The process which lead to that end result as well as the clinical identification of those processes remain areas of considerable controversy. However, the ability of urologists to use sophisticated pressure flow data, or to calculate detrusor energy or power, and in so doing select patients in an early stage of obstructive uropathy is somewhat limited. Whether these methods are unnecessary, and astute clinical observation alone is satisfactory, remains to be conclusively proven, although some workers have suggested, on the basis of reasonably good evidence that this is indeed the case. Patients studied urodynamically and clinically, however, and compared are by the nature of the study somewhat selected, and while carefully observed, they are studied for a relatively short time when compared to the time course of the most common cause of obstructive uropathy, prostatic hypertrophy.

\section{Decentralization and Obstruction}

In addition to commonly accepted syndromes of obstructive uropathy, a type of bladder outlet obstructive phenomenon occurs following parasympathetic bladder decentralization. In this circumstance, the normal neural mechanism which facilitates a change in urethral behavior from an organ of continence, to a compliant conduit does not exist [11], and the decentralized bladder is obstructed by the resting strength of its sphincter [12].
This is a relatively simple system, and an appropriate one for the study of detrusor behavior in response to a relatively fixed outlet resistance. If one considers a situation, in which the motor and sensory pathway from the bladder to the sacral cord (and vice versa) is lost, and the pudendal nerve eliminated e.g. after complete $S_{1}$ to $\mathrm{S}_{4}$ sacral rhizotomy, the equation is reduced to its most basic form. The pressure in the bladder must equal the pressure in the urethra at the time of urinary loss across the urethra. While this situation is relatively rare clinically, it does occur, but is more easily created in the laboratory primate. Measurement of peak urethral closing pressure alone provides information on the maximum bladder pressure attainable, since the pressure regulator is residual urethral sphincter activity. It has been demonstrated that parasympathetic decentralization results in altered storage behavior, with a steeper than normal pressure volume curve. Since the neural mechanism for opening of the "internal sphincter" requires pelvic nerve activity which is lost, urethral pressure remains stable, and the bladder simply fills until intrinsic pressure is equal to urethral resistance at which time leakage occurs. The decentralized bladder becomes trabeculated with an increase in mural thickness, apparently due to muscle hypertrophy, as well as collagen deposition $[9,16]$. The urecholine supersensitivity test becomes markedly positive and with rapid cystometry peculiar contractile wave forms emerge which have diagnostic significance [16].

The magnitude of the bladder pressure has a direct influence on the upper urinary tract in this circumstance. In children, urethral closing pressures higher than 40 are almost always associated with ureteral dilatation and the development of vesicoureteral reflux. It has been demonstrated that bladder pressure has a direct (probably hydraulic) effect on ureteral resistance to perfusion, even in the absence of vesicoureteral reflux which probably explains this phenomenon [10].

Treatment by intermittent catheterization is usually effective, apparently because it reduces detrusor pressure, and sphincter ablative procedures can be shown to have the same effect. In some circumstances the administration of alpha blocking agents and anti- 
cholinergic agents in conjunction with intermittent catheterization are required to decrease detrusor pressure and permit recovery of normal upper urinary tract function. These responses raise questions about the role of neural receptors in the pathogenesis of the syndromes of obstructive uropathy after parasympathetic denervation and in more common clinical expression of detrusor obstructive conditions [13]. It is the combination of anticholinergic therapy and intermittent catheterization in the treatment of parasympathetic denervation which has improved overall outcome, but whether the altered slope of the pressure volume curve with loss of normal storage activity is solely the result of the neural lesion, or represents a response to obstruction has not been determined.

\section{Detrusor Responses to Obstruction with Detrusor Reflex Activity}

Obstructive uropathy is largely but not exclusively a disease of males and includes posterior urethral valves, bladder neck obstruction, prostatic hypertrophy, urethral stricture disease, and rarely meatal stenosis. Reflexly determined detrusor external sphincter dyssynergia, and volitional detrusor sphincter dyssynergia are dissease processes common to males and females. In patients without reflexly determined obstructive syndromes associated with spinal cord injury, symptoms are usually frequency, urgency, slow stream, and often postvoid dribbling. Whether these are due to the obstruction itself, or to the bladder response to obstruction, is not at present clear.

\section{Measurable Changes in Detrusor Function with Obstruction}

Bladder instability occurs in about $50-75 \%$ of patients with prostatic obstruction $[1,2,18]$. This cannot be defined as simply a $15 \mathrm{~cm} \mathrm{H}_{2} \mathrm{O}$ increase in intravesical pressure however, since abnormalities of detrusor "tone" and the viscoelastic properties of the bladder wall due to fibrosis can result in a steep pressure volume curve, which is not the same condition as an uninhibitable reflex bladder contraction despite the definition of the International Continence Society. In order to be certain that a reflex bladder contraction has occured, and thus detrusor instability, some measure of the behavior of the urethral sphincter is essential. A reflex detrusor pressure rise is always accompanied by a change in urethral behavior, while an abnormal pressure volume curve (or decreased compliance) is associated with fixed sphincteric resistance.

Bradley and coworkers were able to eliminate detrusor instability in a small group of patients with prostatic obstruction by the injection of local anesthetic into the prostatic neural plexis [4]. Whether this anesthetic blockade and the abolition of unstable detrusor dysfunction was a result of the block of a hypothetical irritative sensory focus in the prostate itself, as the authors suggested has not been proven. Appell showed that detrusor instability associated with obstructive prostatism usually resolved gradually after transurethral prostatic resection [2]. As most urologists know however, that is not universally the case, but since we have as yet no universally applicable method to exclusively diagnose obstructive uropathy and because detrusor instability is relatively common in patients in the prostatic age group (women as well as men) it is possible that detrusor instability persistent after transurethral resection, represents a primary rather than secondary problem. One of the more difficult diagnostic problems in urology, is the decision in an elderly male, as to whether irritative symptoms with detrusor instability have an obstructive or a neurogenic basis. The voiding profile technique described by Yalla and associates appears to be a valid method for this differential diagnosis [19].

\section{Changes in the Pressure Flow Relationship}

Griffiths, Schaefer, Coolsaet and others have demonstrated the relationship between detrusor contractile activity, flow rate and urethral resistance $[5,8,14]$. Schaefer found detrusor work for a single voiding a relatively fixed value [14]. Changes in pressure were explained by the inverse relationship between flow and urethral resistance, and in the model constructed, were found to be passive rather than active. That is, an increase in pressure was related to an increase in urethral resistance rather than to a compensatory response on the part of the detrusor musculature. Coolsaet and Awad found however, that flow data were not descriptive of obstruction in $40 \%$ of patients who did well after transurethral resection, indicating that perhaps some compensatory activity does take place early in an obstructive process $[3,5]$.

Moreover, it seems likely that the adverse effects of obstructive uropathy on upper tract function, are related to increased pressure associated with the obstruction. The results of pressure flow studies, and detrusor power studies, do however, show that elevated pressures are related in part to urethral resistance and are possible even with a severely damaged bladder, which would be incapable of emptying even if it were to be unobstructed.

\section{Changes in Morphology, Collagen Content and Receptor Activity}

Bladder outlet obstruction leads to increased collagen deposits within the bladder wall $[7,17]$. It has been suggested that collagen deposition impairs smooth muscle 
contractile function, and alters the viscoelastic properties of the bladder wall. Clinically, upper urinary tract deterioration and impairment in renal function have been related to raised intravesical pressures throughout the micturition cycle, or diminished compliance, with increased intravesical pressure during filling, as well as high voiding pressures [6].

Poor compliance with elevated storage phase pressures due to collagen deposition, has been found to be reversible if the obstruction is relieved early, in experimental studies. Trabeculation appears to corralate with collagen deposition, rather than muscular hypertrophy, although both of these processes appear to occur in obstructed bladders. It is not clear what the sequence of hypertrophy and collagen deposition is, and some of the discrepancies in the findings related to the presence or absence of muscular hypertrophy and collagen deposition may reflect the stage of development of detrusor changes in relation to obstruction. Collagen deposition however, beyond a certain point seems unlikely to be influenced by treatment. Myogenic factors are also involved in the pressure volume curve during the storage phase of bladder activity and these could theoretically be altered by stretch and or pharmacologic manipulation. There are certain parallel findings between the parasympathetically denervated detrusor and the obstructed detrusor, which may be related to collagen deposition, which occurs in both instances, but may also be related to myogenic factors which are influenced by neural receptor activity. These appear to be involved in both instances. Susset found abnormal denervation type waves present in patients with benign prostatic hypertrophy by rapid cystometry [15]. In patients with persistent ureteral dilatation after ablation of urethral valves, it was early assumed that the ureterovesical junction obstruction was secondary to a thickened bladder wall. However, renal scanning with lasix washout and Whitaker perfusion testing in these patients usually shows the dilated ureter to be unobstructed. Recently, it has been appreciated that the underlying problem here, is frequently a lack of detrusor compliance, which can be remedied either by augmentation cystoplasty but, also in some instances by the administration of anticholinergic agents and by alpha adrenergic blocking agents. While alpha blocking agents clearly influence vesical outlet behavior, that does not appear to be the sole explanation of their activity in this circumstance, since the most notable effect is not a diminution in voiding pressure, but a return of the pressure volume curve during storage toward normal. Two recent studies have shown that intravesical pressure has a direct effect on ureteral resistance to slow perfusion which in turn suggests that the most valuable characteristic of lower urinary tract function, is maintenance of a flat pressure volume curve during urine storage. In this respect, it is inaccurate and misleading to ascribe a $15 \mathrm{~cm} \mathrm{H}_{2} \mathrm{O}$ pressure increase during bladder filling to detrusor instability. That may be the cause, but disordered compliance does precisely the same thing to the pressure volume curve. Investigation and treatment of conditions which alter compliance is certainly dissimilar to the investigation and treatment of those conditions which lead to detrusor instability.

\section{References}

1. Andersen JT (1976) Detrusor hyperreflexia in benign intravesical obstruction. J Urol 155:532

2. Appell RA (1981) Bladder instability in patients with prostatic hypertrophy. Postgrad Med J 57:640

3. Awad SA (1981) Value and limitations of uroflometry in prostatism. Proceedings of the American Urologic Association, Boston (abstr no 297)

4. Chalfin SA, Bradley WE (1982) Etiology of detrusor hyperreflexia in patients with intravesical obstruction. J Urol 127:938

5. Coolsaet BLRA (1983) Detrusor energy factors. In: Hinman F Jr (ed) Benign prostatic hypertrophy, chap 42. Springer, New York Heidelberg Berlin, p 441

6. George NJR, O'Reilly PH, Barnard RJ, Blackloch NJ (1983) High pressure chronic retention. Br Med J 286:1780

7. Gosling JA, Dixon JS (1983) Detrusor morphology in relation to bladder outflow obstruction and instability. In: Hinman F Jr (ed) Benign prostatic hypertrophy, chap 66. Springer, New York Heidelberg Berlin, p 667

8. Griffiths DJ (1973) The mechanisms of the urethra and of micturition. Br J Urol 45:497

9. Kondo A, Susset JG (1974) Viscoelastic properties of bladder II: comparative studies in normal and pathologic dogs. Invest Urol $11: 459$

10. McGuire EJ, Savastano J, Morrissey S (1983) Bladder and ureteral pressure relationships in non-human primates. J Urol $130: 374$

11. McGuire EJ, Wagner $F$ (1977) The effects of sacral denervation on bladder and urethral function. Surg Gynecol Obstet 114:343

12. McGuire EJ, Woodside JR, Borden TA (1984) Upper urinary tract deterioration in patients with myelodysplasia and detrusor hypertonia: a follow-up study. J Urol

13. McGuire EJ, Woodside JR, Borten TA, Weiss R (1980) Prognostic value of urodynamic testing in myelodysplasia. J Urol 126:206

14. Schaefer W (1976) Eine physiologische Methode zur Beschreibung der Druck-flow-Beziehung während der Miktion. Biomed Tech (Berlin) 21:11

15. Susset JG (1983) Effects of aging and prostatic obstruction on detrusor morphology and function. In: Hinman F Jr (ed) Benign prostatic hypertrophy, chap 65 . Springer, New York Heidelberg Berlin, p 653

16. Susset JG, Peters N, Cohen S, Ghoneim GM (1982) Early detection of neurologic bladder dysfuntion caused by protruded lumbar disc. Urology 20:461

17. Susset JG, Servot-Viguire D, Lamy F, Madernus P, Black R (1978) Collagen in 155 human bladders. Invest Urol 16:204

18. Turner-Warwick RT (1979) Observations on the function and dysfunction of the sphincter and detrusor mechanisms. Urol Clin North Am 6:13

19. Yalla SV (1983 Urethral static pressure profile. In: Hinman F Jr (ed) Benign prostatic hybertrophy, chapt 56 . Springer, New York Berlin Heidelberg, p 577 